\title{
Time series data analysis and ARIMA modeling to forecast the short-term trajectory of the acceleration of fatalities in Brazil caused by the corona virus (COVID-19)
}

\author{
Akini James ${ }^{\text {Corresp., } 1}$, Vrijesh Tripathi ${ }^{1}$ \\ 1 Department of Mathematics and Statistics, Faculty of Science and Technology, University of the West Indies St. Augustine, Port of Spain, Trinidad and \\ Tobago \\ Corresponding Author: Akini James \\ Email address: akini.james1@my.uwi.edu
}

Objective. This paper incorporates the concept of acceleration to fatalities caused by the coronavirus in Brazil from time series data beginning on 17th March 2020 (the day of the first death) to $3^{\text {rd }}$ February 2021 to explain the trajectory of the fatalities for the next six months using confirmed infections as the explanatory variable. Methods. Acceleration of the cases of confirmed infection and fatalities were calculated by using the concept of derivatives. Acceleration of fatality function was then determined from multivariate linear function and calculus chain rule for composite function with confirmed infections as an explanatory variable. Different ARIMA models were fitted for each acceleration of fatality function: the de-seasonalized Auto ARIMA Model, the adjusted lag model, and the auto ARIMA model with seasonality. The ARIMA models were validated. The most realistic models were selected for each function for forecasting. Finally, the short run six-month forecast was conducted on the trajectory of the acceleration of fatalities for all the selected best ARIMA models. Results. It was found that the best ARIMA model for the acceleration functions were the seasonalized models. All functions suggest a general decrease in fatalities and the pace at which this change occurs will eventually slow down over the next six months. Conclusion. The decreasing fatalities over the next six-month period takes into consideration the direct impact of the confirmed infections. There is an early increase in acceleration for the forecast period, which suggests an increase in daily fatalities. The acceleration eventually reduces over the six-month period which shows that fatalities will eventually decrease. This gives health officials an idea on how the fatalities will be affected in the future as the trajectory of confirmed COVID-19 infections change. 


\title{
Time series data analysis and ARIMA modeling to forecast the short-term trajectory of the acceleration of fatalities in Brazil caused by the corona virus (covid-19)
}

\author{
Akini James ${ }^{1}$, Vrijesh Tripathi ${ }^{1}$ \\ ${ }^{1}$ Department of Mathematics and Statistics, Faculty of Science and Technology, University of \\ the West Indies, St. Augustine, Trinidad and Tobago, West Indies \\ ${ }^{2}$ Department of Mathematics and Statistics, Faculty of Science and Technology, University of \\ the West Indies, St. Augustine, Trinidad and Tobago, West Indies \\ Corresponding Author: \\ Akini James ${ }^{1}$ \\ Department of Mathematics and Statistics, Faculty of Science and Technology, University of the \\ West Indies, St. Augustine, Trinidad and Tobago, West Indies \\ Email address: akini.james@yahoo.com
}

\section{Abstract}

Objective. This paper incorporates the concept of acceleration to fatalities caused by the coronavirus in Brazil from time series data beginning on 17th March 2020 (the day of the first death) to $3^{\text {rd }}$ February 2021 to explain the trajectory of the fatalities for the next six months using confirmed infections as the explanatory variable.

Methods. Acceleration of the cases of confirmed infection and fatalities were calculated by using the concept of derivatives. Acceleration of fatality function was then determined from multivariate linear function and calculus chain rule for composite function with confirmed infections as an explanatory variable. Different ARIMA models were fitted for each acceleration of fatality 
31 function: the de-seasonalized Auto ARIMA Model, the adjusted lag model, and the auto ARIMA

32 model with seasonality. The ARIMA models were validated. The most realistic models were

33 selected for each function for forecasting. Finally, the short run six-month forecast was conducted

34 on the trajectory of the acceleration of fatalities for all the selected best ARIMA models.

35 Results. It was found that the best ARIMA model for the acceleration functions were the

36 seasonalized models. All functions suggest a general decrease in fatalities and the pace at which

37 this change occurs will eventually slow down over the next six months.

38 Conclusion. The decreasing fatalities over the next six-month period takes into consideration the

39 direct impact of the confirmed infections. There is an early increase in acceleration for the forecast

40 period, which suggests an increase in daily fatalities. The acceleration eventually reduces over the

41 six-month period which shows that fatalities will eventually decrease. This gives health officials

42 an idea on how the fatalities will be affected in the future as the trajectory of confirmed COVID-

4319 infections change.

\section{Introduction}

\section{Background}

46 The coronavirus (COVID-19) outbreak caused by a virus known as severe acute respiratory

47 syndrome coronavirus 2 (SARS-CoV-2), originated in Wuhan, China and as of $30^{\text {th }}$ June 2020 ,

48 there have been 10.6 million confirmed cases, 5.8 million recovered cases, and 513 thousand

49 deaths recorded worldwide. On $30^{\text {th }}$ June 2020 , Brazil recorded the $2^{\text {nd }}$ highest number of

50 - confirmed cases of the coronavirus in the world and the $2^{\text {nd }}$ highest number of fatalities in

51 the world. This record shows that Brazil had 1.4 million confirmed cases, 790 thousand

52 recovered cases and 59 thousand deaths from the coronavirus (World Health Organization,

53 2020). 
54 During the pandemic, various countries have implemented stringent strategies in their fight

55 against the virus. Since the novel coronavirus started to spread across the world, governments

56 have issued lockdowns, stay-at-home orders, and curfews to help contain the outbreak. However,

57 in Brazil, there was a more relaxed approach from the country's president which in turn has

58 affected the spread of the virus (McLaughlin, 2020).

59 Statement of the Problem

60 To assess the possible future of the pandemic, in particular the fatalities, it is helpful to look not

61 just at the number of cases, but also at how quickly they are increasing (Cohn et al., 2020). Most

62 research focuses on modelling the changes in the death toll due to the coronavirus by observing

63 its behavior based on socioeconomic and demographic factors and not necessarily on the impact

64 of the confirmed infections.

\section{Objective of Study}

66 This paper incorporates the concept of acceleration on the fatalities caused by the coronavirus in

67 Brazil from time series data beginning from $17^{\text {th }}$ March 2020 (the day of the first death) to $3^{\text {rd }}$

68 February 2021 to explain the trajectory of fatalities for the next six months using the confirmed

69 infections as the explanatory variable, that is, the impact of confirmed infections on the number

70 of deaths. Thereafter, forecasting is done to observe the behavior of deaths based on the number

71 of confirmed infections over the next six months.

\section{Velocity and Acceleration}

73 Velocity is the rate of change of fatalities which is the first order derivative of the number of

74 deaths per day (Utsunomiya et al., 2020; Chen and Yu, 2020). However, measuring the speed of

75 the fatalities provides little information about the acceleration in the fatalities. The acceleration

76 functions are much more sensitive and can be better utilized to provide useful information in real 
77 time to monitor, evaluate and forecast the COVID-19 epidemic in Brazil (Chen \& Yu, 2020).

78 Acceleration is the slope of the velocity as a function of time, which is the second derivative. It

79 explains how rapid the change in velocity of fatalities is (Muncaster, 1993). Multivariate linear

80 models as well as the calculus chain rule for composite functions were used to explain the

81 acceleration of fatalities with respect to acceleration of confirmed infections. The chain rule

82 gives us a way to calculate the derivative of a composite function. The chain rule principles state

83 that if fatalities and confirmed infections are two functions then to get the derivative (velocity) of

84 the composite function (death as a function of confirmed cases) simply divide the derivative of

85 fatalities by the derivative of confirmed infections. Similarly, this theory is applied to derive the

86 acceleration function by dividing velocity functions (Nykamp, 2020).

87 Auto Regressive Integrated Moving Average (ARIMA)

88 The Auto Regressive Integrated Moving Average (ARIMA) time series model is a reliable

89 method frequently used in data analysis to forecast times series data and as a result, the

90 acceleration of fatality functions can be used to produce ARIMA models for forecasting

91 purposes. ARIMA models are used for non-stationary data and is made up of the Auto

92 Regression model, $\operatorname{AR}(p)$, which uses the dependent relationship between $Y_{t}$ and $p$ number of

93 lagged observations included in the model, also called the lag order. The model also includes the

94 Integrated (I) aspect, which is the differencing of raw observations, $d$ times to allow for the time

95 series to become stationary. Making the time series data stationary is necessary since stationary

96 series are relatively easier to predict. Most statistical forecasting is based on the fact that the

97 times series can be approximately stationarized through the use of mathematical transformations.

98 The predictions for the stationarized series can then be "untransformed" by reversing whatever

99 mathematical transformations used, to obtain predictions for the original series (Nau, 2020). If a 
100 time series dataset has a stable long-run trend and tends to revert to the trend line following a

101 disturbance, it may be possible to stationarize it by de-trending (removing the effects of trends).

102 However, in such a dataset like the corona virus in Brazil, sometimes even de-trending is not

103 sufficient to make the series stationary, in which case it may be necessary to transform it into a

104 series of period-to-period and/or season-to-season differences. That is, differencing the time

105 series. Stationarizing a time series through differencing (where necessary) is an important part of

106 the process of fitting an ARIMA model. The difference order, $d$, is the differences between

107 consecutive observations (Kenton, 2020). The last aspect of the ARIMA model includes the

108 Moving Average model MA( $q$ ) which uses the dependency between $Y_{1 t}$ and a residual error

109 from a moving average model applied to $q$ lagged observations. Hence the time series model is

110 denoted as ARIMA (p,d,q) model (Chen, 2019; Stock and Watson, 2007;Box. et al., 2016).

\section{Purpose of the Study}

112 The acceleration of fatality ARIMA models were used for forecasting the pandemic to come to a

113 reasonable understanding of the trajectory of deaths in the short run. Short-term forecasts

114 generated can be useful to guide the allocation of resources that are critical to bring the epidemic

115 under control (Roosa et al. 2020).

\section{Review of Literature}

117 Throughout the period of the pandemic, numerous studies have been conducted to better analyze

118 and make predictions regarding the end of the pandemic. Researchers have developed models to

119 make forecast about the fatalities due to the coronavirus.

120 Researchers have used the count of daily cases to formulate ARIMA models to predict the end of

121 the pandemic (Bayyurt and Bayyurt, 2020; Dehesh, Mardani-Fard and Dehesh, 2020). One

122 study adopted three kinds of mathematical models, that is, logistic model, Bertalanffy model and 
123 Gompertz model where the logistic model was the best fit among the three models. In this

124 research, the epidemic trends of SARS were first fitted and analyzed to prove the validity of the

125 existing mathematical models. The results were then used to fit and analyze the situation of

126 COVID-19. This is the case since COVID-19 and SARS virus are both variants of coronaviruses

127 and the infection pattern may be similar (Jai et al., 2020). The logistic model was used to explore

128 the risk factors and predict the probability of occurrence of the disease according to the risk

129 factors. It can predict the development and transmission law of epidemiology.

130 Many other study designs have made predictions based on compartmental models, with the

131 population divided into classes and with assumptions being made about the rate of transfer from

132 one class to another. These models are mostly differential equation prediction models. This

133 employed mathematical modeling techniques to study the transmission and spread of COVID-19

134 to predict the magnitude and timing of the epidemic peak and the final epidemic size under

135 various intervention strategies. Carcione et al. (2020) used the Susceptible-Exposed-Infectious-

136 Removed (SEIR) model to describe the spread of the virus and compute the number of infected

137 and dead individuals. This model aimed to compute the number of infected, recovered, and dead

138 individuals based on the number of contacts, probability of disease transmission, incubation

139 period, recovery rate and fatality rate. Okhuse (2020) used a similar approach to propose a

140 mathematical model for the end in the spread and subsequent elimination of the virus by using a

141 new deterministic endemic model (Susceptible-Exposed-Infectious-Removed-Undetectable-

142 Susceptible: SEIRUS). This study combined quarantine observatory procedures and behavioral

143 change social distancing in the control and eradication of the disease in the most exposed sub-

144 populations. 
145 Veloso and Ziviani (2020) conducted a study by modelling the country level death toll velocity

146 and acceleration. They used factors such as the daily COVID-19 death toll curve in each country,

147 country's countermeasures in response to the COVID-19 pandemic, community mobility reports,

148 estimations of critical care beds available for and needed by COVID-19 patients in each country,

149 and country's development indicators.

150 Another researcher conducted a study on the velocity and acceleration, utilizing the concept of

151 motion to observe the confirmed cases in China to explain the spread of the epidemic. This was

152 then compared to the spread after massive interventions that took place in China (Chen and $Y u$,

153 2020).

154 Most studies used socioeconomic and demographic factors to explain the pandemic and

155 thereafter, make projections based on these factors. However, this study seeks an alternative

156 approach, by observing the confirmed infections since it is highly correlated to the fatalities. It is

157 assumed that if the number of the confirmed infections can determine the nature of the fatalities,

158 then better predictions can be made about the pandemic. This study borrows the concept of

159 motion used by Chen and $\mathrm{Yu}$ (2020) and applies it to data from Brazil. This study does not make

160 comparisons with the interventions that took place in Brazil to come to conclusions like most of

161 the previously mentioned studies. The derivative functions represent the velocity and

162 acceleration of fatalities based on the velocity and acceleration of confirmed infections,

163 respectively. Our study has used the confirmed infections as the explanatory variable for

164 fatalities. In addition, the concept of time series ARIMA modeling has been used to produce

165 forecasts.

166 Materials \& Methods

$167 \quad$ Data 
168 The data repository for the 2019 Novel Coronavirus was retrieved online from the Center for 169 Systems Science and Engineering (CSSE) at Johns Hopkins University (Dong, Du \& Gardener, 170 2020). The dataset contains data on the cumulative confirmed infections, cumulative recovered 171 cases and the cumulative fatalities in Brazil starting from the $17^{\text {th }}$ of March 2020, the day of the 172 first recorded death. This is 21 days after the first recorded confirmed infection in Brazil. On the $17317^{\text {th }}$ of March 2020, Brazil already had a total of 321 confirmed infections and two recovered 174 cases. The cumulative confirmed infections and cumulative fatalities show an upward trend. The dataset contained the COVID-19 cases for a period of approximately 11 months (46 weeks) in Brazil until the $3^{\text {rd }}$ of February 2021 which is 344 days from the first confirmed infection and

177324 days from the first death in Brazil. At the end of the study period, Brazil had already

178 recorded 9, 339,420 confirmed infections, 8,311,881 recovered cases and 227,563 fatalities.

179 However, there are 799,976 active cases. Therefore, at this point there exist $89 \%$ of the patients

180 recovered, $2.4 \%$ deaths and the remaining $8.6 \%$ still active cases.

181 Velocity and acceleration of the confirmed infections and fatalities

182 Time series data on the cumulative confirmed infections and cumulative fatalities were observed 183 where each $x_{i}$ and $z_{i}$ are the daily confirmed infections and daily fatalities, respectively, for $i=$ $184(21,22, \ldots t) . i=21$ is the $21^{\text {st }}$ day since the first recorded confirmed infection which is the day of 185 the first recorded fatality in Brazil. Equation (1) below shows the general equation for the 186 cumulative confirmed infections and cumulative fatalities.

187

$$
Y(y)=\int_{i=21}^{t} y_{i}=\sum_{i=1}^{t} y_{i}
$$
where $Y(y)=\{F(x)$ confirmed infections, $H(z)$ confirmed fatalities $\}$ 
190 However, $F(x)$ and $H(z)$ being extremely insensitive to changes in the pandemic, the first

191 derivatives of equation (1) produces the general function, $Y^{\prime}(y)$, in equation (2) (Chen and $Y u$, 192 2020). These functions are said to be velocity functions which explains the change in the 193 confirmed infections and change in fatalities caused by the pandemic in Brazil. That is, the new 194 cases each day. This explains the speed of the confirmed infections and fatalities for $i=(21,22$, $195 \ldots t) . i=21$ is the $21^{\text {st }}$ day since the first recorded confirmed infection which is the day of the 196 first recorded fatality in Brazil.

197

$$
Y^{\prime}(y)=\int_{i=21}^{t+1} y_{i}-\int_{i=21}^{t} y_{i}=\sum_{i=21}^{t+1} y_{i}-\sum_{i=21}^{t} y_{i}
$$

where $Y^{\prime}(y)=\left\{F^{\prime}(x)\right.$ velocity of confirmed infections, $H^{\prime}(z)$ velocity of fatalities $\}$

Although $F^{\prime}(x)$ and $H^{\prime}(z)$ can measure the speed of the pandemic, they do not give any information regarding the acceleration, which is much more sensitive than the velocity functions 202 (Chen and $Y u, 2020)$. Thus, the second derivatives, $F^{\prime \prime}(x)$ and $H^{\prime \prime}(z)$ produced the general 203 equation (3) below (Utsunomiya et al., 2020). This represents the acceleration of the confirmed 204 infections and acceleration of fatalities each day in Brazil due to the pandemic, respectively 205 (Chen and $Y u, 2020$ ). The acceleration explains how rapid is the change in velocity. If the 206 acceleration functions produce zero, this is an early indication of neither acceleration nor 207 deceleration of the pandemic. Where the acceleration functions produce values greater than zero, 208 it is an early indication of acceleration of the pandemic and producing values less than zero 209 indicates deceleration.

$$
Y^{\prime \prime}(x)=Y^{\prime}\left(x_{i}+1\right)-Y^{\prime}\left(x_{i}\right)
$$
where $Y^{\prime \prime}(y)=\left\{F^{\prime \prime}(x)\right.$ acceleration of confirmed infections, $H^{\prime \prime}(z)$ acceleration of fatalities $\}$ 


\section{Multivariate Linear Fatality Function}

214 Correlation analysis using ggpairs in R Programming software were used to produce the best fit

215 multivariate linear functions from the data produced by the functions (Schloerke et al., 2020).

216 Log transformation was done to meet the linear functions assumptions for normality (Feng et al., 217 2014). These functions were used to explain and forecast the velocity and the acceleration of the 218 fatalities due to the coronavirus.

\section{Velocity of Fatality Composite Functions}

220 Due to the obvious relation the confirmed infections have on the number of fatalities, the

221 velocity of fatality composite functions of confirmed infections was utilized to formulate the rate

222 at which deaths were occurring each day with respect to new confirmed infections named $\frac{d H(z)}{d F(x)}$ as

223 shown in equation (4). This term explains the speed in the deaths influenced by the confirmed

224 infections. The term begins at the date of the first recorded death, $i=21$ which is the $21^{\text {st }}$ day

225 since the first recorded confirmed infection.

$$
\frac{d H(z)}{d F(x)}=H^{\prime}(z) \div F^{\prime}(x) \rightarrow \frac{d H(z)}{d F(x)}=H^{\prime}(z) \times\left[F^{\prime}(x)\right]^{-1}
$$

\section{Acceleration of Fatalities Composite Functions}

229 The derivatives of the above velocity of fatality composite function formed the acceleration of

230 fatality composite function as seen in equation (5). That is, the term $\frac{d H^{\prime}(z)}{d F^{\prime}(x)}$, explains the rate at 231 which the deaths accelerate with respect to the acceleration of the confirmed infections. This 232 rate was used to explain and forecast the acceleration of fatalities caused by the pandemic. The 233 term starts at the date of the first recorded death, $i=21$ which is the $21^{\text {st }}$ day since the first 234 recorded confirmed infection. 


$$
\frac{d H^{\prime}(z)}{d F^{\prime}(x)}=H^{\prime \prime}(z) \div F^{\prime \prime}(x) \rightarrow \frac{d H^{\prime}(z)}{d F^{\prime}(x)}=H^{\prime \prime}(z) \times\left[F^{\prime \prime}(x)\right]^{-1}
$$

236

\section{Time Series Analysis}

238 The data for acceleration of fatality functions were plotted using months as the horizontal axis.

239 Data cleaning is often the first step that data scientists and analysts take to ensure statistical 240 modelling is supported by good data (Hyndman, 2014). Therefore, the behavior of the acceleration 241 of the fatalities within each month were then observed by plotting a monthly breakout of the data 242 for fatality functions. This was done to observe the range of possible outliers within each month. 243 Tsclean function in R programming software was used on the time series data for each of the 244 acceleration of fatality functions to identify and replace any outliers or blanks with estimated 245 values from the time series data using series smoothing and decomposition (Hyndman et al., 2020 246 and Dalinina, 2017). Outliers are residuals that lie outside the range $\pm 2\left(q_{0.9}-q_{0.1}\right)$ where $q_{p}$ is the $247 p$ quantile of the residuals. The residuals are identified by fitting a loess curve for non-seasonal 248 data and via a periodic STL decomposition for seasonal data (Hyndman, 2014). However, this 249 cleaned time series data, now referred to as the cleaned function, was graphed and observed. Only 250 where there were extreme variances and volatility with the clean data, monthly (every 30 days 251 from start) and weekly (every 7 days from start) moving averages (MA) were formed and 252 compared to the cleaned data. Moving average is used to analyze data points by creating a series 253 of averages of different subsets of the full data set to mitigate the impact of random short-term 254 fluctuations over a specified timeframe (Hayes, 2020). That is to smooth out any noise or possible 255 random outliers still present and to emphasize long term trends (Ross, 2019). However, given that 256 the acceleration functions have cyclical patterns at most (bouncing upward and down) moving 257 averages are not likely to capture meaningful trends thus over-smoothing the data (Smith, 2020). 
258 Hence, with the moving averages being too smooth, the cleaned data was used to form ARIMA 259 models to forecast the trajectory of the velocity and acceleration of fatalities. The graphs produced 260 from the two acceleration of fatality functions were plotted using months as the unit on the 261 horizontal axis. Thereafter, the time series data was decomposed by splitting it into three 262 components: seasonality, trends, and remainder. These intuitive components capture the historical 263 patterns in the series and deconstructing a series into these components can help understand its 264 behavior and prepare a foundation for building a forecasting model (Dulinina, 2017). Seasonality 265 refers to patterns that repeat with a fixed period, trends are the underlying trend of the metrics and 266 the remainder also known as the noise is the original time series after the seasonal and trend series 267 are removed (Anomaly, 2020). This was done using the stl function in the $\mathrm{R}$ programming software 268 (Cleveland et al., 1993). Seasonality over time, the trend line and the remainder of the models 269 selected for forecasting in the previous step were observed. ARIMA models can be fitted to both 270 seasonal and non-seasonal data. However, seasonal ARIMA requires a more complicated 271 specification of the model structure. For this cause, we first attempted to de-seasonalize the series 272 and use a non-seasonal ARIMA model (Ghosh, 2020 and Dalinina, 2018). The function seasadj 273 in the R programming software accomplishes this where it returns the seasonally adjusted data 274 constructed by removing the seasonality (Hyndman, 2020).

275 The Augment Dikey Fuller Test (ADF) was then conducted on the selected cleaned data to test for 276 stationarity. The ADF null hypothesis is that the variable has unit root (non-stationary) and the 277 alternative hypothesis is that the variable does not have a unit root (Holmes, Scheuerell \& Ward, 278 2020). If the p-value of the ADF test is small, it concludes stationarity of the data. On the other 279 hand, where the printed p-values were large, we fail to reject the null hypothesis which means that 280 the data is non-stationary (Hua 2016). Stationarity and seasonality of the dataset can be further 
281 analyzed by using autocorrelation function (ACF) and partial autocorrelation function (PACF)

282 graphs (Taspinar, Celebi \& Tutkun, 2012). The cleaned data are plotted by using the ACF and

283 PACF functions in R Programming (Hyndman et al., 2020). This plot shows the correlation

284 between the time series and its lagged value. When the lines are outside the bounds of the ACF

285 and PACF graphs, this suggests serious lags. According to how drastic the lag is, this will suggest

286 non-stationarity. However, with the series being non-stationary, the data will be differenced until

287 stabilization starting with a difference order of one and re-evaluation on whether further

288 differencing is needed. This therefore eliminates (or reduces) trend and seasonality and converts

289 the non-stationary series to a stationary series. Fitting an ARIMA model requires the series to be

290 stationary since modeling a stable series with consistent properties involves less uncertainty.

291 Hence, stationarity is required because only if the time series data is a deterministic (non-random)

292 pattern, the research can use the ARIMA time series model (Hua, 2016). The ADF test is then

293 conducted again but on the differenced data to test for its stationarity. ACF and PACF were plotted

294 on the differenced data and the lags were observed.

295 Fitting the ARIMA Model

296 The auto fit ARIMA model was produced on the de-seasonalized (without seasonality) cleaned

297 data. This function uses a variation of the Hyndman-Khandakar algorithm which combines unit

298 root tests, minimization of the Akaike Information Criterion (AIC) and Maximum Likelihood

299 Estimation (MLE) to obtain an ARIMA model (Hyndman et al.,2020; Hyndman and

300 Khandakar, 2008). The auto fit ARIMA model can produce a forecast, however, it must be

301 checked to see if the model order parameter and structure are correctly specified ensuring that

302 there are no significant autocorrelation present. This was done using the ACF and PACF plots,

303 observing lags and adjusting the $p$ or $q$ to ensure that there are no significant autocorrelations

Peer] reviewing PDF | (2020:10:53390:2:0:NEW 8 Jun 2021) 
304 present. That is, ensuring all lines were within the bounds of the PCF and PACF graphs.

305 Additionally, models were also selected based on the Akaike Information Criterion (AIC). The

306 AIC value for each model was observed and comparisons were made. This is a widely used

307 measure of a statistical model. It basically quantifies the goodness of fit and the simplicity/

308 parsimony, of the model into a single statistic. When comparing models, the one with the lowest

309 AIC is generally "better" (Keshavani, 2013). The AIC was observed and compared amongst the

310 auto ARIMA model and the adjusted model.

311 The adjusted model was validated for each of the functions using the holdout method by dividing

312 the time series data into a training set and a testing set. The training set is what the model is

313 trained on and the testing set is used to evaluate how well the model performs on unseen data

314 (Kapil, 2018). A subset of the dataset (20\%) was omitted from the ARIMA model. That is, a

315 subset of 65 was set out of the dataset starting from case 260 to 324 to be used as the test set to

316 see how well the model performs. It provides a final estimate of the machine learning model's

317 performance after it has been trained and validated. The remaining $80 \%$ of the data (no hold out)

318 were then plotted. This is the training set, and it is what the model will be trained on. The

319 selected ARIMA models for both acceleration functions were then forecasted for the rest of the

320 study period using the forecast function in the R programming software to observe how the $95 \%$

321 and $80 \%$ forecasting interval fits the actual data points (Hyndman et al., 2020). The lines of the

322 time series of the de-seasonalized counts were also plotted to compare the forecast with the

323 actuals. Thereafter, we observe how the $95 \%$ and $80 \%$ forecasting interval fits the actual data

324 points. If the validation shows that the model is not a good one, seasonality on the de-

325 seasonalized cleaned data will be re-introduced and an ARIMA model would be produced on

326 this. A model is not good if the line does not fall within the forecast interval and if the expected 
327 forecasts on the de-seasonalized model is too linear too soon, which is unlikely given the past 328 behavior of the series (Dalinina,2017). The AIC for each model was observed and comparisons 329 were made. Based on this validation, the better ARIMA models for each function were used for 330 forecasting the trajectory of the acceleration of fatalities for the next six months. A 30-day 331 forecast was also done on the ARIMA models for each validated acceleration functions, mainly 332 for comparison and observational purposes amongst the respective models. The ARIMA models 333 for each validated function were the auto ARIMA model without seasonality, the adjusted lag 334 model, and the Auto ARIMA model with seasonality. The most realistic ARIMA model for each 335 validated function was then selected for forecasting the next six-month period.

\section{Results}

\section{Acceleration of Confirmed infections and Fatalities}

338 Figures $1(a)$ and (b) show the acceleration functions $F^{\prime \prime}(x)$ and $H^{\prime \prime}(z)$ derived in equation (3)

339 and are the second derivative of $F(x)$ and $H(z)$, respectively. The acceleration of confirmed

340 infections, $F^{\prime \prime}(x)$, shows continuous fluctuations in acceleration and deceleration where the

341 magnitude of these changes increased until approximately the $119^{\text {th }}$ day since the first confirmed

342 infection (23 June 2020). Thereafter the curve maintained its magnitude until $195^{\text {th }}$ day since

343 the first confirmed infection ( $7^{\text {th }}$ September 2020$)$ until $279^{\text {th }}$ day $\left(30^{\text {th }}\right.$ November 2020$)$. Within

344 such period there were random distinct spikes. The curve then gradually increased in magnitude

345 throughout the rest of the study period, Figl (a).

346 The acceleration of fatalities, $H^{\prime \prime}(z)$, have fluctuations where the horizontal axis represents the

347 number of days from the first fatality, Fig $1(b) . H^{\prime \prime}(z)$, shows fluctuations between the acceleration

348 and deceleration of fatalities about the zero line that gradually increased in magnitude until the

$34998^{\text {th }}$ day since the first confirmed infection ( $2^{\text {nd }}$ of June 2020) where this magnitude was then 
350 maintained until the $203^{\text {rd }}$ day since the first confirmed infection $\left(15^{\text {th }}\right.$ September 2020).

351 Thereafter, the curve decreased in magnitude with few distinct spikes and then gradually increased

352 throughout the rest of the study period.

\section{Multivariate Linear Functions}

354 The distributions and correlations on the raw data produced from the acceleration function in

355 equation (4) were explored. This was done to understand the relationship amongst functions to

356 conduct multivariate linear regressions for the acceleration of fatalities. However, producing

357 linear functions requires the data for all functions to meet the normality assumptions previously

358 mentioned. As a result, $\log$ transformation was done on the functions formed from equation (3)

359 before producing linear functions. Due to the negatives produced in the acceleration function

360 from equations (3) and (4), a common technique was used by adding a constant value to the data

361 before applying the log transformation (Wicklin, 2011). The transformation for the acceleration

362 of confirmed infections and fatalities was, therefore, $\log \left(F^{\prime \prime}(x)+a_{1}\right)$ and $\log \left(H^{\prime \prime}(x)+a_{2}\right)$,

363 respectively where $a_{1}$ and $a_{2}$ are constants. These constants were chosen so that the $\min \left(F^{\prime \prime}(x)\right.$

$\left.364+a_{1}\right)$, and $\min \left(H^{\prime \prime}(x)+a_{3}\right)$ will be a small positive number, 0.001 . As a result, the data was

365 then transformed to $\log \left(F^{\prime \prime}(x)+51285.001\right)$ and $\log \left(H^{\prime \prime}(x)+974.001\right)$. Thus, the distribution

366 and correlation on the transformed data was observed and normality of the functions were

367 checked. The correlation between functions were used to form the multivariate linear function

368 listed below.

369 The following acceleration of fatality linear function were produced using the acceleration of 370 confirmed infections as the explanatory variables:

$$
R=0.9956
$$


374 Death_Acceleration1 function has a coefficient of determination $R^{2}=99.56 \%$. That is, $99.56 \%$

375 of the independent variable explains the acceleration of fatality suggesting that it is a good

376 function.

377 The acceleration of fatality linear function designed in equation (6) was plotted over the study 378 period which is approximately 46 weeks from the $9^{\text {th }}$ to $54^{\text {th }}$ week Fig. 2 . The first week is when 379 the first recorded confirmed infection took place whereas the $9^{\text {th }}$ week is when the first recorded 380 death occurred. Figure 2 shows that the Death_Acceleration1 function initially has a constant 381 acceleration at zero until $1^{\text {st }}$ April 2020, but the curve's magnitude gradually increased until $3^{\text {rd }}$ 382 June 2020 where it maintained this average magnitude until $15^{\text {th }}$ September 2020 . The 383 acceleration started reducing its magnitude thereafter until $24^{\text {th }}$ November 2020 and then started 384 largening throughout the rest of the study period.

385 Acceleration of Fatality Composite Functions

386 Figure 3 shows $\frac{d H^{\prime}(z)}{d F^{\prime}(x)}$, the acceleration of deaths as confirmed infections accelerate, and this 387 function generally fluctuates a little above the zero line with three distinct pulses on 27th March 388 2020, 26th August 2020 and 22nd October 2020 that shows drastic deceleration of the fatalities 389 and then the curve rapidly accelerated, returning to its average nature. The other noticeable 390 pulses on 5th May 2020 and 5th June 2020 show sharp and large acceleration at first with an 391 immediate deceleration back to its average nature.

\section{Explanatory Data Analysis}

393 Breakout of the data 
394 A monthly breakout on the time series data produced from the above acceleration functions was 395 done to observe the range of any possible outliers in the data points. Each of the functions of 396 acceleration showed that there were very few noticeable outliers throughout the study period.

\section{Cleaned Functions and Moving Averages}

398 However, to compensate for any possible outliers, the acceleration functions were first cleaned to 399 produce cleaned functions for each. In addition to such, the weekly moving average (every 7 400 days from the start of the study period) and the monthly moving average (every 30 days from the 401 start of the study period) functions were formed from the cleaned functions. The weekly and 402 monthly moving averages were compared to their respective cleaned functions, and it showed 403 that the moving averages were over-smoothing the data. That is, not capturing meaningful trends. 404 Hence the cleaned functions were used for creating a model for forecasting.

\section{Decomposition of the Data}

406 The cleaned function for each fatality function of acceleration was decomposed by extracting its 407 seasonality, its trend line, and its remainder. Figure 4 shows the decomposition of the acceleration of fatality functions, along with its associated cleaned data where the horizontal axis represents the number of weeks since the first death in Brazil. Seasonality component was extracted from the cleaned data for each of the acceleration of fatality functions called the de-

411 seasonalized data and this de-seasonalized series was used to create ARIMA models.

\section{Stationarity}

413 An ADF Test was firstly done on the cleaned data. This test outputs the Dickey Fuller value, Lag 414 Order, and p-values. The smaller the Dickey Fuller value, the better the model (Holmes,

415 Scheuerell \& Ward, 2020). The Lag Order allows for higher-order autoregressive processes and 416 the p-values conclude whether the model has unit root or not. The cleaned data for all the 
417 functions have small p-values which suggests stationarity of the functions. However, the ACF

418 and PACF functions were used to verify this and lags for each function were observed.

\section{Autocorrelation and Choosing a model}

420 ACF plots of the de-seasonalized series showed significant autocorrelations with many lags. The

421 PACF plots of the de-seasonalized series significant autocorrelations with many lags. The PACF

422 plots showed that this could be due to carry-over correlation from the first lag in most cases.

423 Hence, the de-seasonalized series was differenced until stabilization starting at difference order

424 one. The ADF test was conducted again, on the difference data, and for all functions, it rejects

425 the null hypothesis of non-stationarity. Table 1 shows the ADF test results for each acceleration

426 of fatality functions and their difference order required to obtain stationarity and reduced lags.

427 Both functions now show smaller Dickey Fuller values than the ADF test first conducted on the

428 cleaned data and small p-values. Hence the differenced data is better.

429 Thereafter ACF and PACF were produced for the differenced data for the acceleration functions

430 to observe any spikes at specific lag points of the differenced series. It was found that the lags

431 are not as drastic for the differenced data. This therefore suggests that the differencing of order

432 one for the acceleration of fatality functions is sufficient and should be included in the model for

433 each function.

\section{Fitting the ARIMA model}

435 Different models were fitted for each of the acceleration of fatality functions. The models are the 436 Auto ARIMA Model without seasonality, a custom made ARIMA model called the adjusted lag 437 model and the Auto ARIMA model without seasonality. The adjusted lag model compensates for 438 the serious lags found, if any, in the auto ARIMA model without seasonality. Table 2 shows the 
439 fitted ARIMA models for the acceleration functions with their respective AIC values. The

440 smaller the AIC value the better the model.

441 Cross Validation: Holdout Method

442 The Adjusted lag models were validated using the holdout method showing that the actual data

443 were all within the $95 \%$ and $80 \%$ forecast interval which shows that it is a good model. Fig. 5 .

444 However, the blue lines representing forecasts for the adjusted model for $\frac{d H^{\prime}(z)}{d F^{\prime}(x)}$ function seems

445 unrealistic since the blue lines representing forecast in this function seems as though it might

446 become linear too soon. That is the case since plotted predictions assume that there will be no

447 other seasonal fluctuations in the data and the change in number of fatalities from one day to

448 another is constant in mean and variance. Hence this forecast may be a naive model. As a result,

449 seasonality was added back to this series and its predictions were also observed using the holdout

450 method.

451 The horizontal axes in Fig. 5 represent time in days. The vertical axes for the acceleration of

452 fatality graphs represents the acceleration of fatality rates per acceleration of confirmed

453 infections. The interpretation of the vertical axes gives little information due to the many

454 manipulations of the data. As a result, we focus on the trajectory of the forecasts.

455 Further Testing, Selecting the Best Model, Forecasting and Analyzing

456 A further testing was done by observation, comparing a 30-day forecast on the ARIMA models

457 listed in Table 2 for each of the acceleration functions; Death_Acceleration 1 and $\frac{d H^{\prime}(z)}{d F^{\prime}(x)}$.ARIMA

458 models were selected based on how realistic the model looked over the 30-day forecast. The

459 better ARIMA model selected for the acceleration functions were the auto ARIMA model with

460 seasonality. This is confirmed for $\frac{d H^{\prime}(z)}{d F^{\prime}(x)}$ where the auto ARIMA model with seasonality has the 
461 lowest AIC value. However, the decision made for the Death_Accelerationl function goes

462 against the AIC value which would have suggested that the adjusted lag model is better for

463 making forecasts. Based on the 30-day forecast for such a model, the predicted curve was too

464 constant, and its forecast interval started expanding like a funnel which seems a bit unrealistic

465 when compared to the auto ARIMA model with seasonality. The summary on selecting the best

466 ARIMA model for each function is summarized. Fig. 6.

467 The six months forecasted ARIMA models for the multivariate linear function for the

468 multivariate acceleration of fatalities function shows an increase and then a constant trajectory

469 until where the magnitude of the acceleration gradually decreases over the forecasted period.

470 Fig. 7(a)). However, the composite function for the acceleration of fatalities shows large

471 fluctuations at first but then gradually decreases in magnitude until it seems to eventually flatten.

472 Fig. 7(b).

\section{Discussion}

474 This study incorporates the concept of acceleration of fatalities caused by the coronavirus in

475 Brazil to explain the projected trajectory of fatality for the next six months using the confirmed

476 infections as the explanatory variable.

477 Two different functions were used to explain the acceleration of fatalities using the acceleration

478 of the confirmed infections as the explanatory variable. One of the accelerations of fatality

479 function is a multivariate linear function, Death_Acceleration 1 and the other function, $\frac{d H^{\prime}(z)}{d F^{\prime}(x)}$, was

480 formed in equation (5) by applying calculus chain rule for composite functions to produce the

481 acceleration of fatality composite function.

482 Acceleration of Cases, Multivariate Functions and Composite Functions 
483 The acceleration in the confirmed infections generally increased in magnitude throughout the 484 period. The acceleration eventually maintained an average range of values. Fig. 1 (a). Closer 485 observation showed that when the magnitude of the acceleration of the fatalities decreased over 486 the period, the speed in the fatalities (velocity) slowed down. Hence, how rapid these fatality 487 cases are occurring daily affects the outcome of the number of cases. Moreover, a close 488 relationship can be seen between the confirmed infections and the fatalities since there exist 489 similarities in their curves throughout the study period.

490 When the acceleration got smaller in magnitude, the change in fatalities started to reduce. On the 491 other hand, where the speed in the change of the fatalities (acceleration) increased in magnitude, 492 the change in the fatalities (velocity) started to increase. Although these functions consider the 493 confirmed infections to explain the outcome of the fatalities, by observation, it shows to be a 494 good representation of the actual fatalities' functions produced from the raw data.

495 The multivariate function shows that the magnitude in acceleration increased over the period but 496 eventually maintained in magnitude. Fig 2. The composite function shows that the average size 497 of the acceleration of the fatalities was basically small and constant throughout the period. Fig. 3. 498 These functions developed in Fig. 2 and Fig. 3 seem to mirror the general results of the actual 499 cases represented in Fig. 1(b).

\section{ARIMA Modelling of Functions and Forecasting}

501 The acceleration of fatality functions had very few distinct outliers throughout the study period.

502 Nevertheless, the time series data for each function was still cleaned from any possible outliers 503 and missing data points. Moving averages were expected to be taken on each of the cleaned 504 functions to produce ARIMA models for forecasting. This would have worked well for our 505 model since in reality new confirmed infections or changes in new confirmed infections at one 
506 point in time do not have an immediate impact on fatality and change in fatality, respectively.

507 The moving averages provides for historical information. However, the moving averages for the

508 acceleration of fatality functions are far smoother than its cleaned data. Using these moving

509 averages can result in erroneous forecasts due to the data being too smooth which can cause a

510 loss in information. Hence the cleaned functions produced were used instead for the ARIMA

511 modelling to make forecasts.

512 The ARIMA models produced for each of acceleration of fatality functions are shown in Table 2.

513 After conducting a cross validation (the holdout method) on the adjusted lag models and further

514 testing of the ARIMA models by forecasting for the next 30 days, both acceleration models were

515 found to be realistic to forecast for the next six months. Fig. 5. The forecast in the acceleration

516 composite function initially shows a large fluctuation in the acceleration function which suggests

517 a quickening in daily fatalities. Thus, an increase in the velocity of fatalities in the earlier part of

518 the six-month forecasted period is expected. However, the acceleration started to decrease which

519 mean that the rate at which fatalities were changing would eventually start to reduce. This model

520 was replicated for São Paulo, one of the 26 states of the Federative Republic of Brazil. This state

521 was chosen since it was the first state to record a Covid19 death in Brazil (Albuquerque, 2020).

522 The forecast shows some similarities to the forecast made on the country Brazil where there

523 exists some fluctuation in the beginning of the period until eventually flattening throughout the 524 rest of the study period. Fig. 8 .

525

\section{$526 \underline{\text { Limitations }}$}

527 This study had several limitations. There exists little referencing regarding the conclusions of

528 this research since most journals did not forecast the accelerations of fatalities in Brazil by using 
529 confirmed infections as an explanatory variable. Also, there is little interpretation to the values in

530 the $y$-axis for the forecast of the acceleration curves due to the many manipulations of the data.

531 As a result, only the trajectory of the forecast was observed over time.

\section{Conclusions}

533 In general, the increased acceleration of fatalities in the earlier part of the forecasted period

534 suggests an increase in daily fatalities. The increasing numbers in fatalities in Brazil during

535 February 2021 and March 2021 confirms this prediction of increased fatalities as suggested by

536 the acceleration model in this paper (Worldometer, 2021). It is expected by the model that the

537 reduction in acceleration of fatalities will cause the new fatalities to slow down thus causing an

538 eventual decrease in the fatalities over the next six-month period.

\section{References}

540 Albuquerque F. 2020, Brazil reports first death from Covid-19. AgenciaBrasil. Available at

541 Brazil reports first death from Covid-19|Agência Brasil (ebc.com.br). (accessed 06 June 2021).

542 Anomaly. 2020. Extracting Seasonality and Trend from Data: Decomposition Using R-

543 Anomaly. Available at https://anomaly.io/seasonal-trend-decomposition-in-r/index.html

544 Bayyurt L, Bayyurt B. 2020. Forecasting of COVID-19 Cases and Deaths Using ARIMA

545 Models. https://doi.org/10.1101/2020.04.17.20069237

546 Box GEP, Jenkins GM, Reinsel GC, Ljung GM.2016. Time Series Analysis: Forecasting and

547 Control $5^{\text {th }}$ Edition. John Wiley \& Sons, Inc.

548 Carcione JM, Santos JE, Bagaini C and Ba J. 2020. A Simulation of COVID-19 Epidemic

549 Based on a Deterministic SEIR Model. Font. Public Health 8:230.doi:10.3389/fpubh.2020.00230

550 Chan CH, Chan GCH, Leeper TJ, and Becker J. 2018. rio: A Swiss-army knife for data file

$551 \quad$ I/O. R package version 0.5 .16 
552 Chen J. 2019. Autoregressive Integrated Moving Average Investopedia.

553 https://www.investopedia.com/terms/a/autoregressive-integrated-moving-average-arima.asp

554 Chen X., Yu B. 2020. First two months of the 2019 Coronavirus Disease (COVID-19) epidemic

555 in China: real-time surveillance and evaluation with second derivative model. Journal of Global

556 Health Research and Policy. DOI: 10.1186/s41256-020-00137-4

557 Cleveland RB, Cleveland WS, McRae JE, Terpenning I. 1993. STL: A Seasonal-Trend

558 Decomposition Procedure Based on Loess. Journal of Official Statistics, 6, 3-73.

559 Cohn N, Katz J, Sanger-Katz MS, Quealy K.2020. Four Ways to Measure Coronavirus

560 Outbreaks in U.S. Metro Areas. The New York Times.

561 https://www.nytimes.com/interactive/2020/03/27/upshot/coronavirus-new-york-comparison.html

562 (assessed 3 September 2020).

563 Dalinina R. 2017. Introduction to Forecasting with ARIMA in R. Available at Introduction to

564 Forecasting with ARIMA in R $\mid$ Oracle Data Science (accessed 1 January 2020).

565 Dehesh T, Mardani-Fard H.A, Dehesh P. 2020. Forecasting of COvID-19 Confirmed Cases in

566 Different Countries with ARIMA Models. https://doi.org/10.1101/2020.03.13.20035345

567 Dong E, Du H, Gardener L. 2020. An interactive web-based dashboard to track COVID-19 in 568 real time. The Lancet Infectious Diseases.533-534.https://doi.org/10.1016/S1473-

$5693099(20) 30120-1$

570 Feng C, Wang H, Lu N, Chen T, He H, Lu Y, Tu XM. 2014. Log transformation and its

571 implications for data analysis. Shanghai Archives of Psychiatry Vol 26, No.2 105-109 DOI:

$572 \quad 10.3969 /$ j.issn.1002-0829.2014.02.009

573 Gardener M. 2019. Statistics for Ecologists Edition 2. Available at Logistic regression model

574 prediction. Statistics for Ecologists Exercises. (dataanalytics.org.uk) (accessed 26 January 2020). 
575 Ghosh B. 2018. Advanced Modeling in R Forecasting with ARIMA - Part 1. Available at

576 Forecasting with ARIMA - Part I| DataScience+ (datascienceplus.com) (accessed 19 January

577 2020).Hayes A. 2020. Moving Average (MA) Definition Investopedia. Available at

578 https://www.investopedia.com/terms/m/movingaverage.asp

579 Holmes EE, Scheuerell MD, Ward EJ. 2020. Applied Time Series Analysis for Fisheries and

580 Environmental Data. Northwest Fisheries Science Center,2725 Montlake Blvd E., Seattle, WA 58198112.

582 Hua Y. 2016. The Relationship between Public Expenditure on Education and Economic

583 Growth: Evidence from China. All Theses.2361. https://tigerprints.clemson.educ/all theses/2361

584 Hyndman R. 2014. New in Foreast 5.0. Hynsight. Available at New in forecast $5.0 \mid$ Rob J

585 Hyndman (accessed on 24 May 2021).

586 Hyndman R, Athanasopoulos G, Bergmeir C, Caceres G, Chhay L, O'Hara-Wild

587 M,Petropoulos F, Razbash S, Wang E, Yasmeen F. 2020._forecast: Forecastingfunctions for

588 time series and linear models_. R package version 8.12,

$589<$ URL:http://pkg.robjhyndman.com/forecast>.

590 Hyndman, RJ, Khandakar, Y. 2008. Automatic time series forecasting: The forecast

591 package for R. Journal of Statistical Software, 27(1),1-

592 22. https://doi.org/10.18637/jss.v027.i03

593 Adrian Trapletti and Kurt Hornik. 2019. tseries: Time Series Analysis and Computational

594 Finance. R package version 0.10-47

595 Jia L, Li K, Jiang Y, Guo X, zhao T.2020. Prediction and analysis of Coronavirus Disease

596 2019. https://arxiv.org/abs/2003.05447 
597 Johns Hopkins University. 2020. COVID-19 Data Repository by the Center for Systems

598 Science and Engineering (CSSE) at Johns Hopkins University. Available at

599 https://github.com/CSSEGISandData/COVID-19 (accessed on 16 September 2020).

600 Kapil AR. 2018. Application in Python, Model Evaluation and Validation, Holdout Cross-

601 Validation. Available at https://www.datavedas.com/holdout-cross-validation/ (accessed on 8

602 September 2020).

603 Keshvani A. 2013. Using AIC to Test ARIMA Models. Available at Using AIC to Test ARIMA

604 Models - CoolStatsBlog. (accessed 25 January 2020).

605 Kourentzes N. 2016. How to choose a forecast for your time series. Available at

606 https://kourentzes.com/forecasting/2016/06/17/how-to-choose-a-forecast-for-your-time-series/

607 (accessed16 September 2020)

608 Muncaster R. 1993. A-Level Physics, Fourth Edition. United Kingdom: Nelson Thornes Ltd.,2-3

609 McLaughlin, K. 2020. Brazil is letting the coronavirus run wild with little intervention, and the

610 results are strikingly bad. Business Insider. Available at https://www.businessinsider.com/brazil-

611 coronavirus-no-nationwide-lockdown-results-are-grim-2020-5. (accessed 30 June 2020)

612 Nau R.2020. Statistical forecasting: notes on regression and time series analysis. Available at

613 Statistical forecasting: notes on regression and time series analysis (duke.edu). (accessed 26

614 January 2020)

615 Nykamp DQ. 2020. “The idea of the chain rule.” From Math Insight.

616 http://mathinsight.org/chain_rule_idea

617 Okhuese VA.2020.Mathematical predictions for COVID-19 as a global pandemic.

618 doi: https://doi.org/10.1101/2020.03.19.20038794.

619 https://www.medrxiv.org/content/10.1101/2020.03.19.20038794v1 
620 Roosa K, Lee Y, Luo R, Kirpich A, Rothenberg R, Hayman JM, Yan P, Chowell G. 2020.

621 Short-term Forecasts of the COVID-19 Epidemic in Guangdong and Zhejiang, China: February

622 12-23, 2020. Journal of Clinical Medicine 9:596-607 DOI:10.3390/jcm9020596

623 Ross S. 2020. Why is the Moving Average (MA) Important for Traders and Analysts?

624 Investopedia. Available at https://www.investopedia.com/ask/answers/122314/why-moving-

625 average-ma-important-traders-and-analysts.asp

626 Schloerke B, Cook D, Larmarange J, Briatte F, Marbach M, Thoen E, Elberg A and

627 Crowley J. 2020. GGally:Extension to 'ggplot2'. R package version 2.0.0. https://CRAN.R-

628 project.org $/$ package $=$ GGally

629 Smith K. 2020. The 7 Pitfalls of Moving Averages Investopedia. Available at

630 https://www.investopedia.com/articles/trading/11/pitfalls-moving-averages.asp

631 Stock JH, Watson MW. 2007. Introduction to Econometrics 2nd Edition. Pearson Education

632 Inc. 535-539

633 Taspinar F, Celebi N, Tutkun N. 2012. Forecasting of daily natural gas consumption on regular

634 basis in Turkey using various computational methods. Journal of Elsevier Energy and Buildings

635 56. 23-31. DOI: 10.1016/j.enbuild.2012.10.023

636 Utsunomiya YT, Utsunomiya ATH, Torrechilha RBP, Paulan SC, Milanesi M, Garcia JF.

637 2020. Growth and Acceleration Analysis of the COVID-19 Pandemic Reveals the Effect of

638 Public Health Measures in Real Time. Frontiers in

639 Medicine.7:247.DOI:10.3389/fmed.2020.00247

640 Veloso A. Ziviani N. 2020. Explainable death toll motion modeling: COVID-19 narratives and

641 counterfactuals. DOI: 10.1101/2020.07.04.20146423 
642 Wickham H, Averick M, Bryan J, Chang W, McGowan LD, François R, Grolemund G, 643 Hayes A, Henry L, Hester J, Kuhn M, Pedersen TL, Miller E, Bache SM, Müller K, 644 Ooms14 J, Robinson D, Seidel DP, Spinu V, Takahashi K, Vaughan D, Wilke C, Woo K 645 ,Yutani H. 2019. Welcome to the tidyverse. Journal of Open Source Software, 4(43), 1686, 646 https://doi.org/10.21105/joss.01686

647 Wicklin, R. 2011. Log transformation: How to handle negative data values? The Doo Loop. 648 Available at blog.sas.com (accessed 25 June 2020).

649 World Health Organization, 2020. WHO Corona Virus Disease (COVID-19) Dashboard.

650 Available at https://covid19.who.int/region/amro/country/br (accessed 30 June 2020).

651 Worldometer.2021. Brazil Coronavirus. Available at Brazil Coronavirus: 12,324,765 Cases and 652 303,726 Deaths - Worldometer (worldometers.info). (assessed 3 September 2021). 
Figure 1

\section{Acceleration}

(A) Confirmed Infections (B) Fatalities
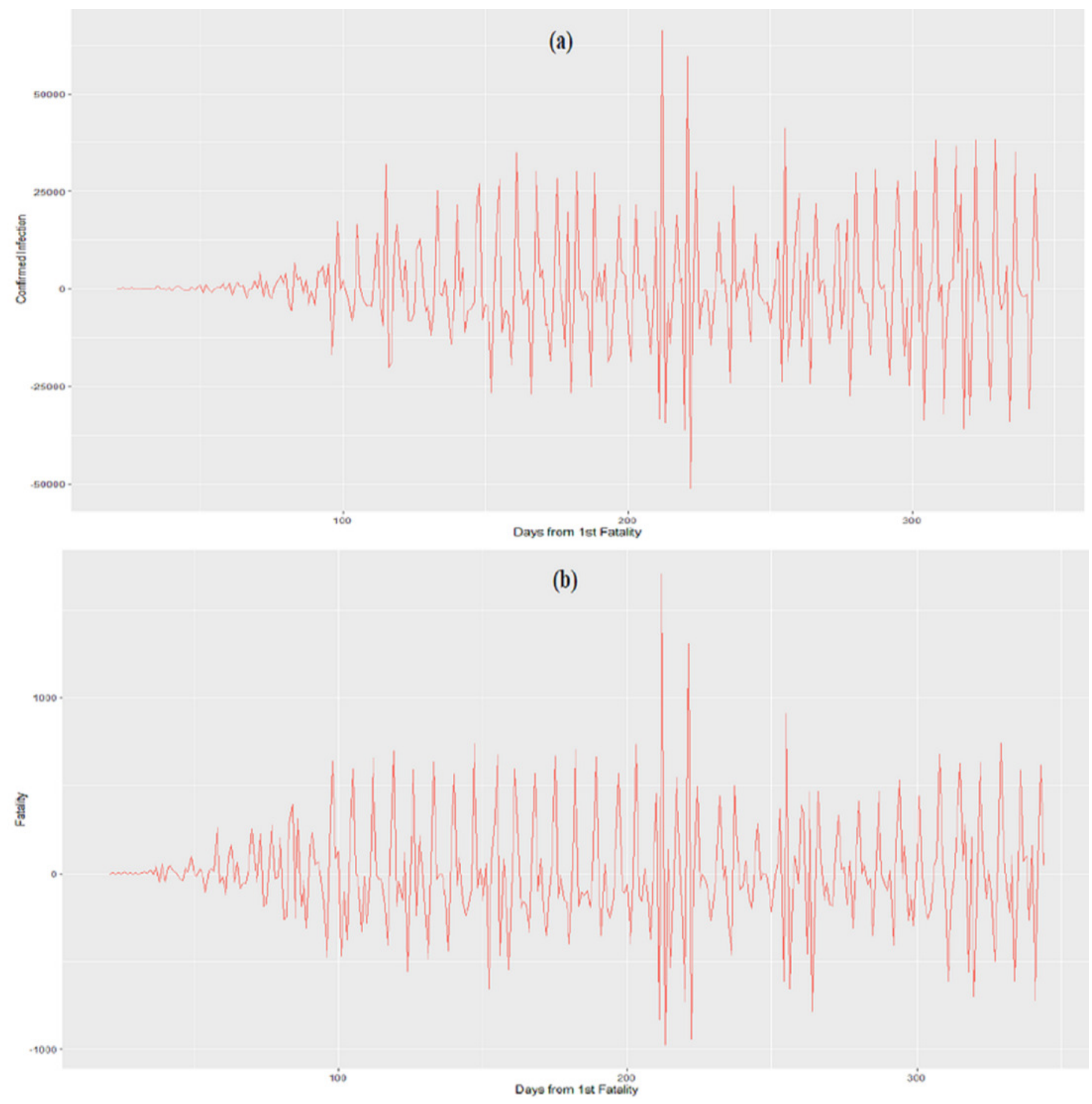
Figure 2

Multivariate Linear Functions for Death_Acceleration1

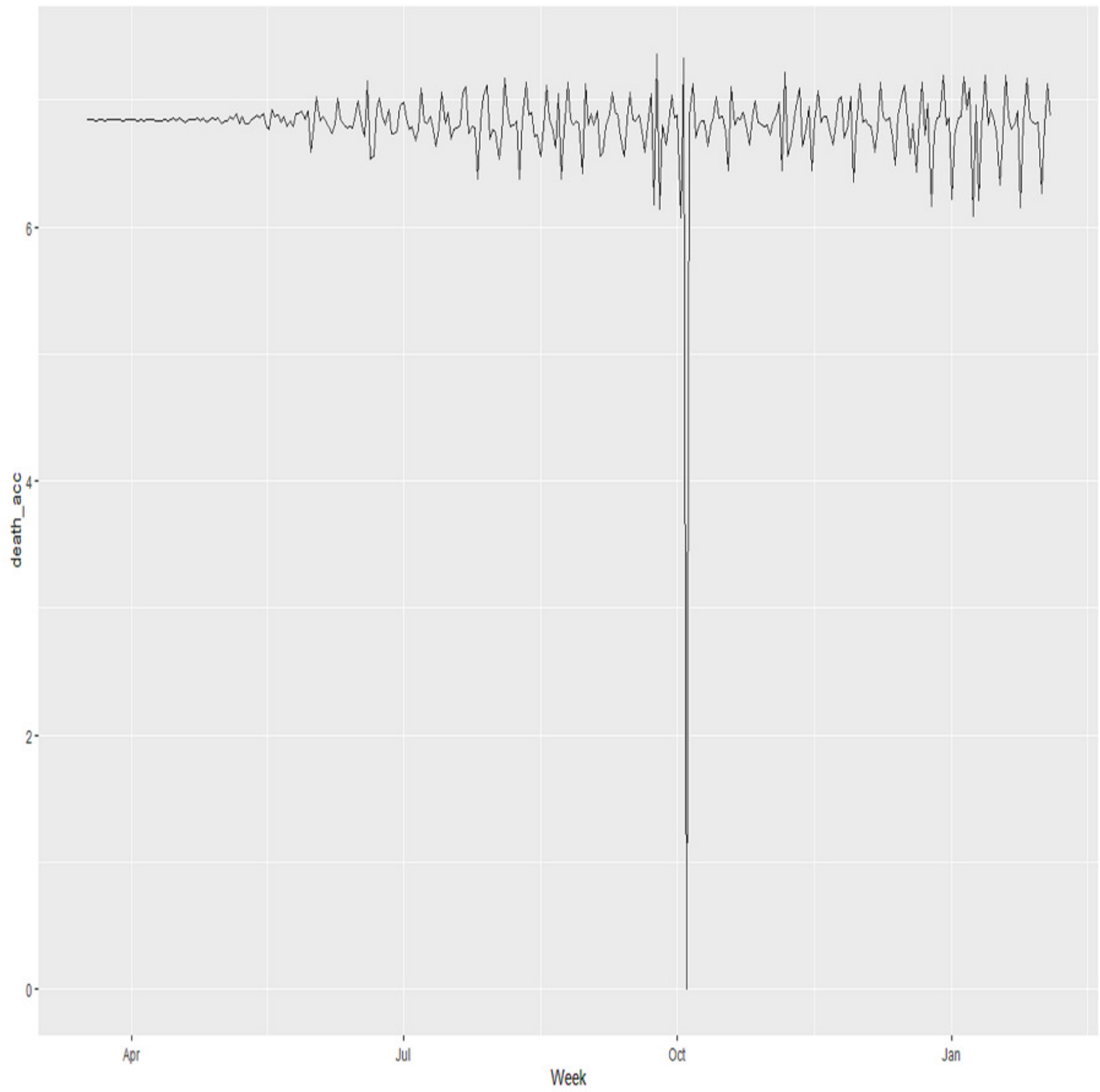


Figure 3

Fatality Composite Functions for the acceleration in Fatality with respect to the acceleration of Confirmed infections

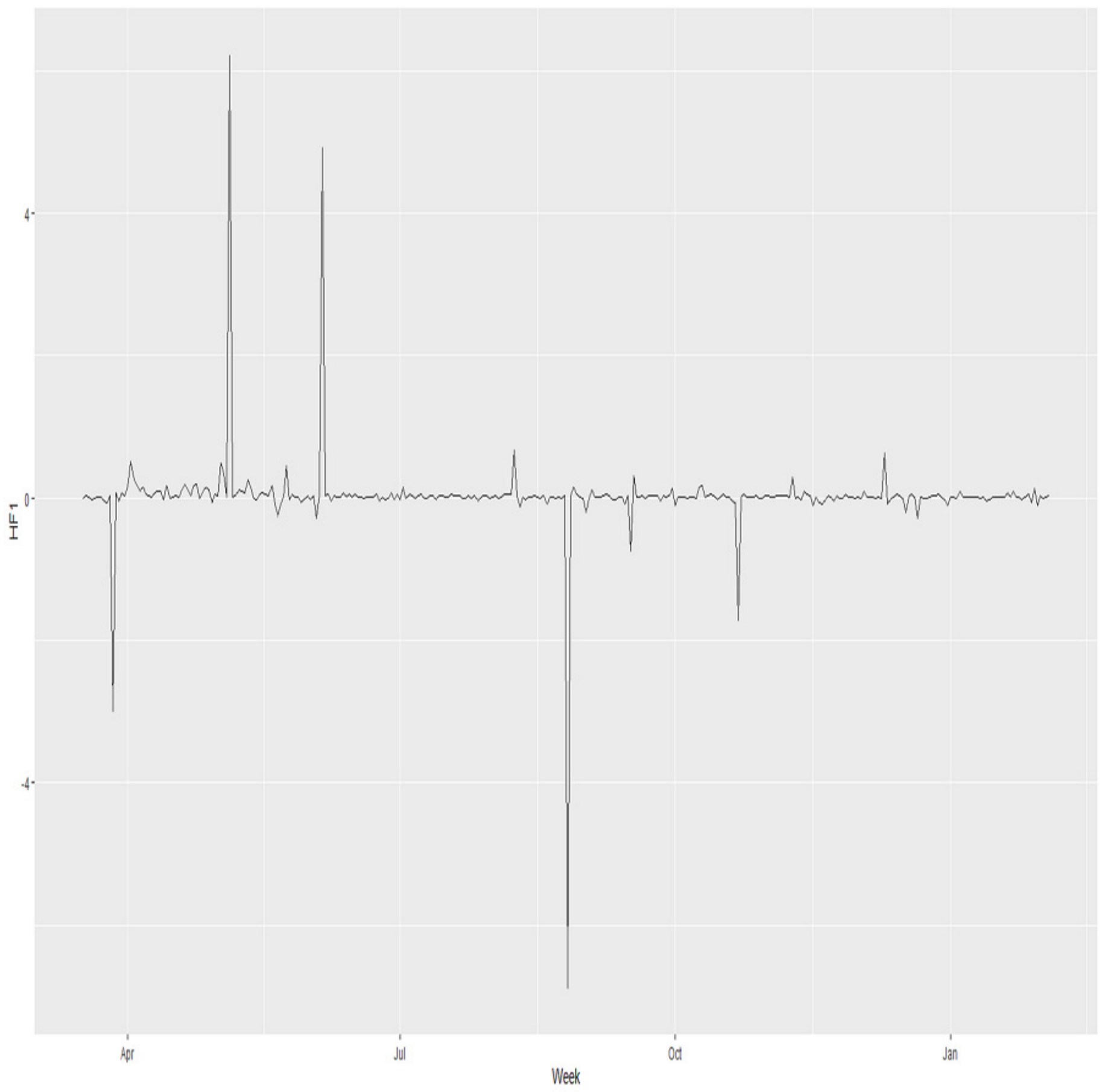


Figure 4

Decomposition of the cleaned data acceleration of Fatality

(a) cleaned data for Death_Acceleration1 (b) cleaned data for composite function with respect to the change confirmed infections. 


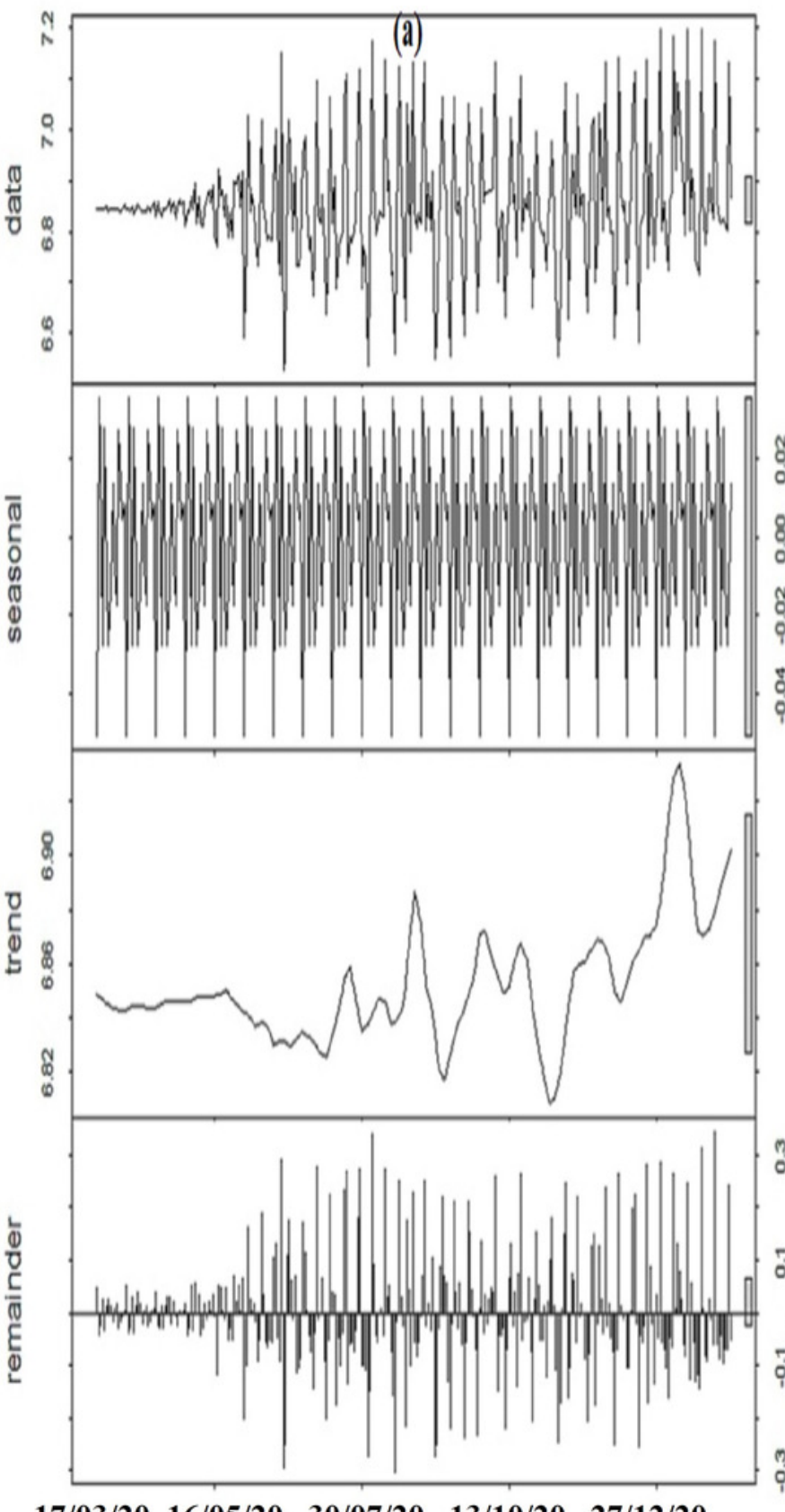

$\begin{array}{lllll}17 / 03 / 20 & 16 / 05 / 20 & 30 / 07 / 20 & 13 / 10 / 20 & 27 / 12 / 20\end{array}$

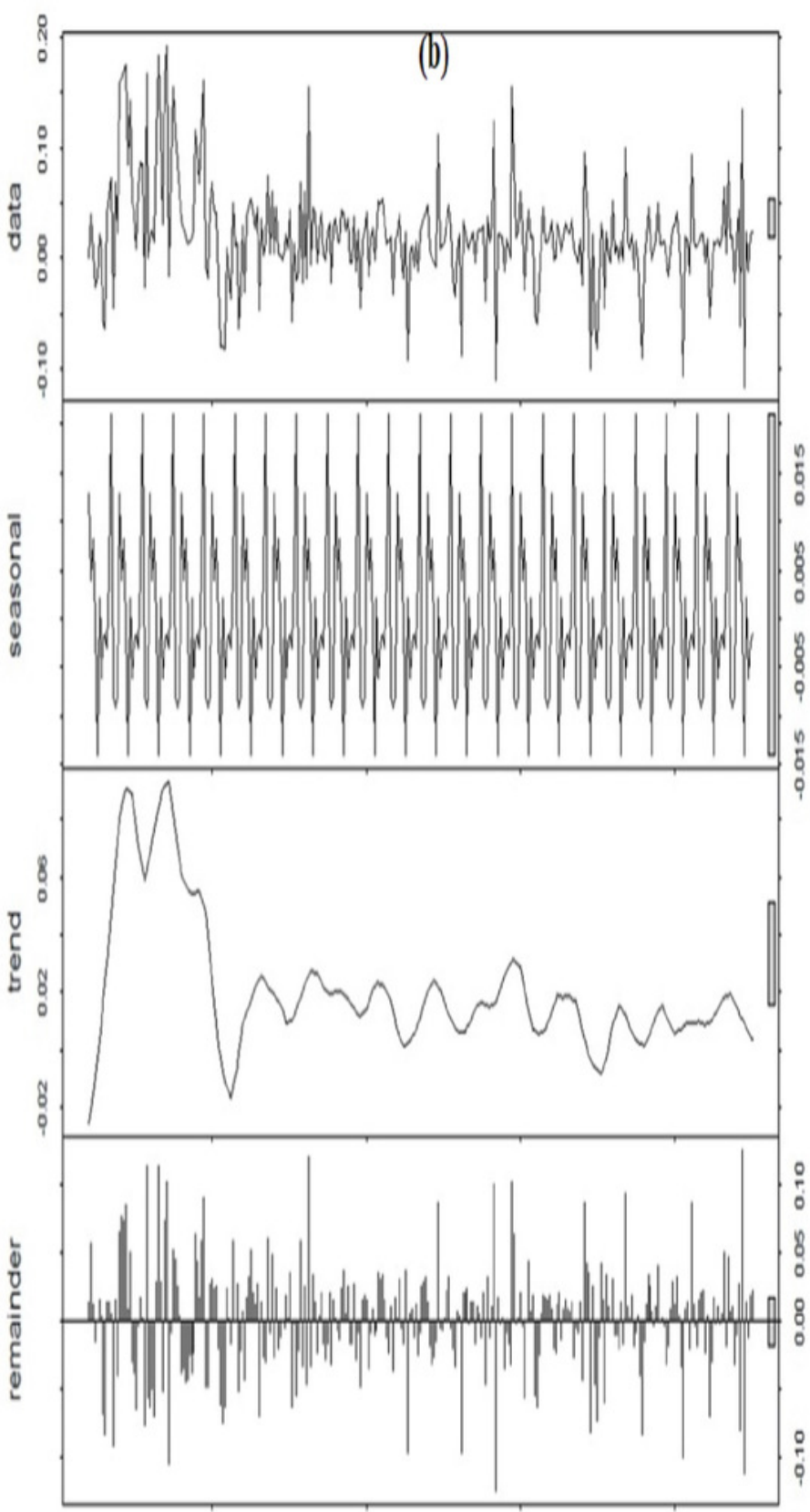

$\begin{array}{lllll}17 / 03 / 20 & 16 / 05 / 20 & 30 / 07 / 20 & 13 / 10 / 20 & 27 / 12 / 20\end{array}$

time 
Figure 5

Cross Validation using Holdout Method for Acceleration of Fatalities

(a) Death_Acceleration1 (b) Composite function with respect to the change in confirmed infections. 

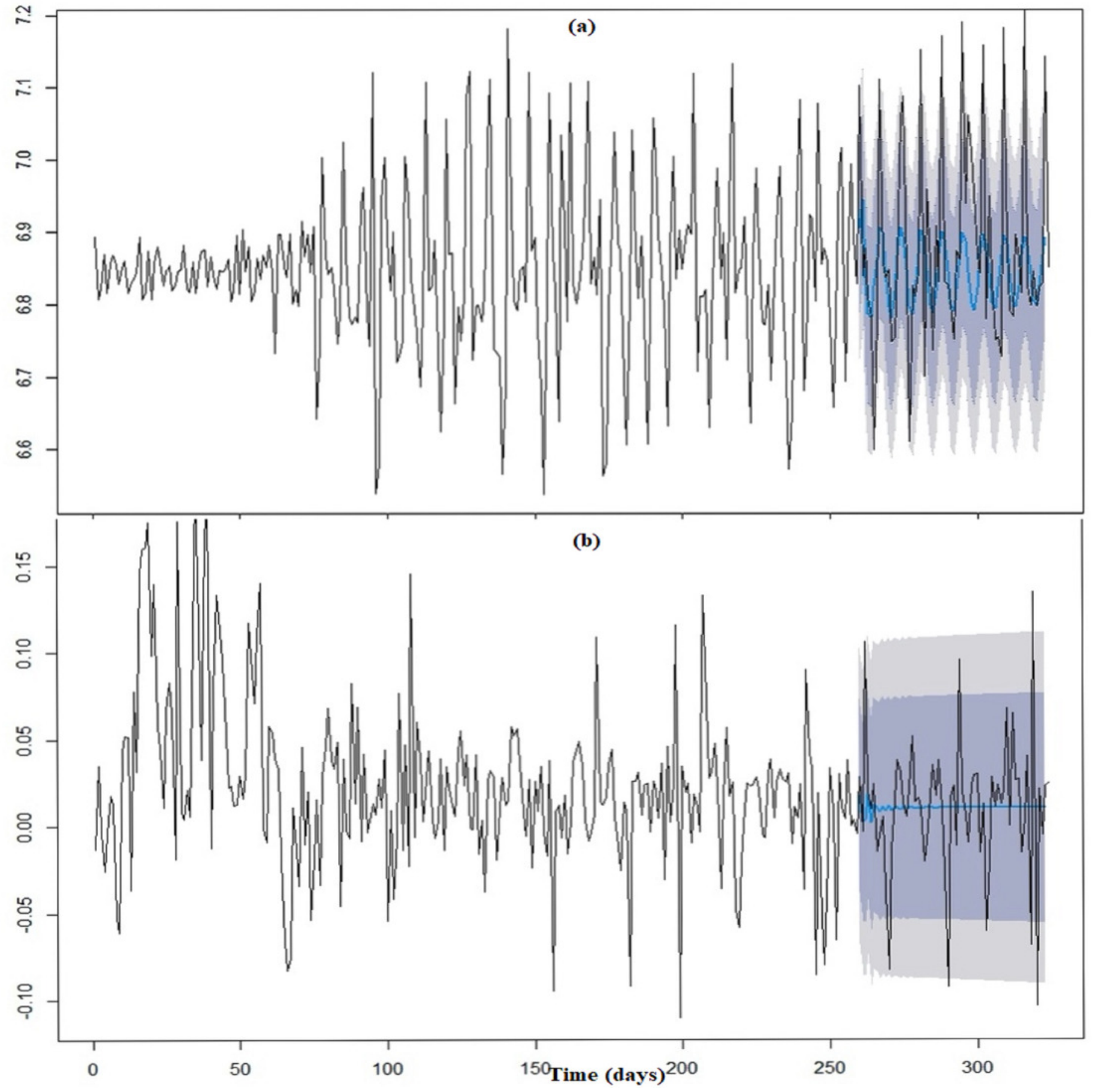
Figure 6

Acceleration of Fatalities validated function for forecasting sequence diagram.

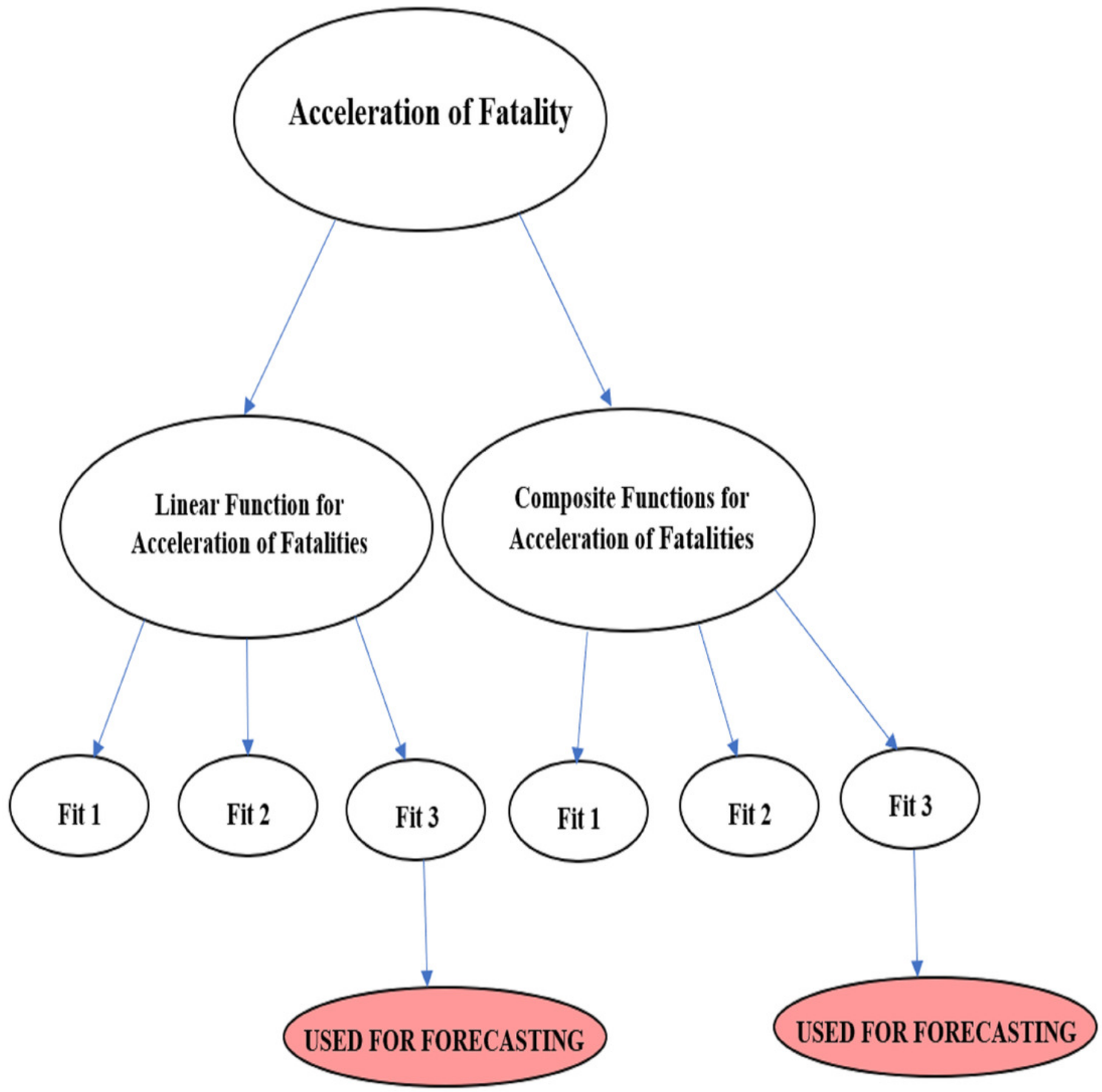


Figure 7

Seasonalized Auto ARIMA Model Six Months' Forecast of Acceleration of Fatalities

(a) Death_Acceleration1 (b) Composite function with respect to the change in confirmed infections. 

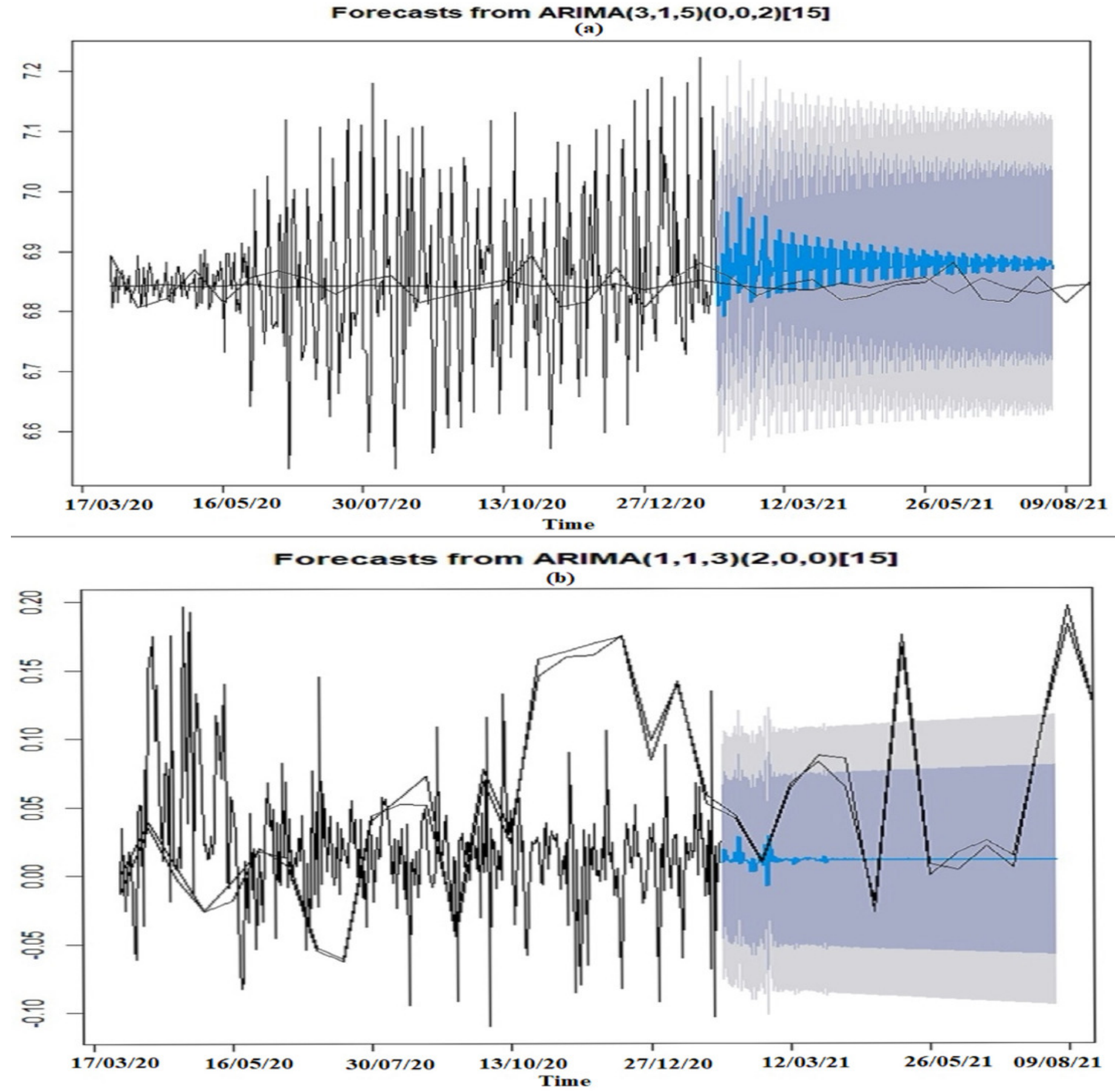


\section{Figure 8}

Six Months' Forecast of Acceleration of Fatalities for São Paulo

(a) Multivariate Function (b) Composite Function.
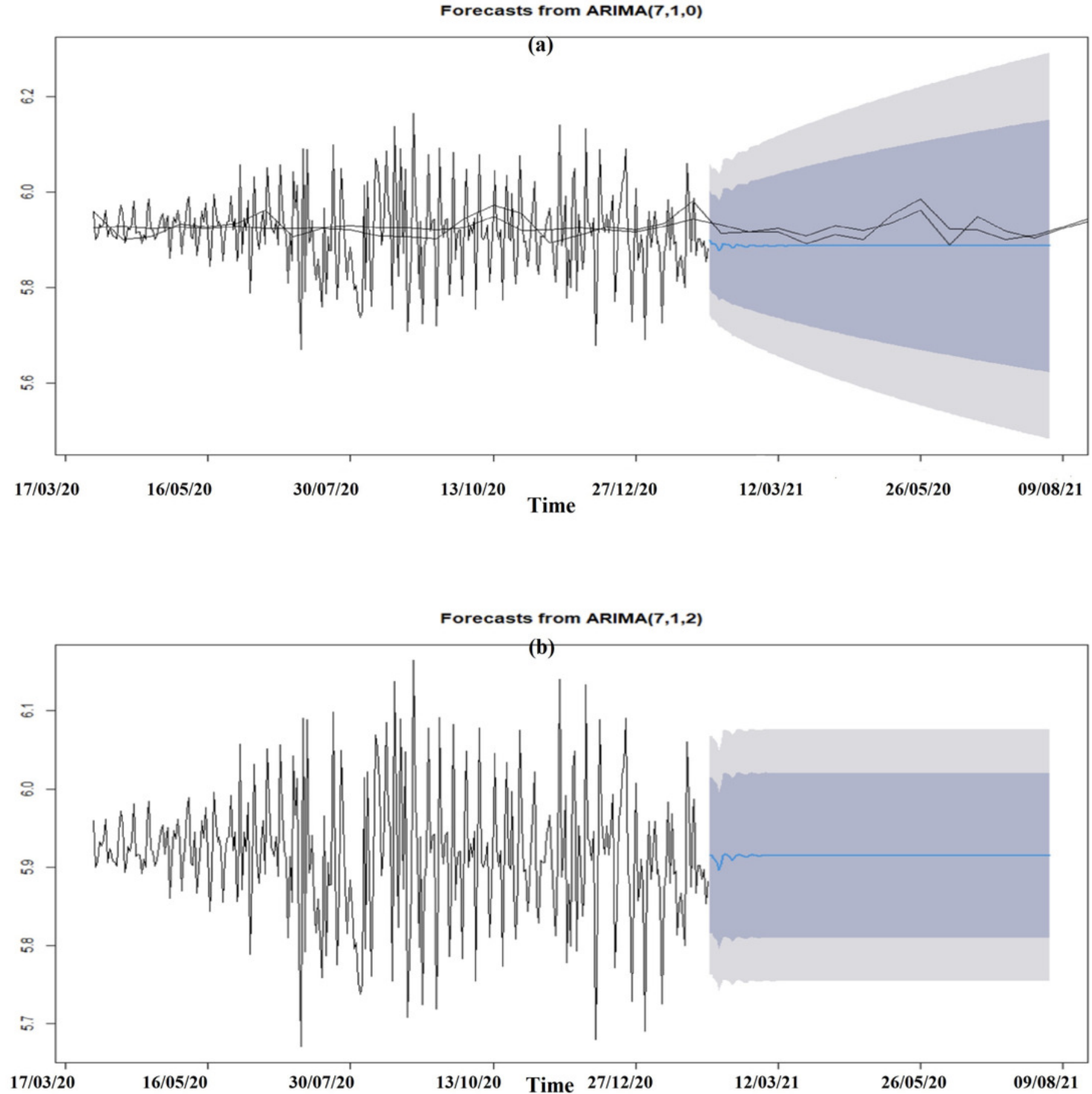


\section{Table $\mathbf{1}$ (on next page)}

Augmented Dickey Fuller (ADF) Test for Differenced Acceleration Fatality Functions 
1

\begin{tabular}{|c|l|l|l|l|}
\hline & $\begin{array}{l}\text { Difference } \\
\text { Order }\end{array}$ & Dickey Fuller & Lag Order & $\begin{array}{l}\text { p- } \\
\text { value }\end{array}$ \\
\hline Death_Acceleration1 & 1 & -14.488 & 6 & 0.01 \\
\hline$\frac{\boldsymbol{d} \boldsymbol{H}^{\prime}(\mathbf{z})}{\boldsymbol{d} \boldsymbol{F}^{\prime}(\boldsymbol{x})}$ & 1 & -9.6114 & 6 & 0.01 \\
\hline
\end{tabular}


Table 2 (on next page)

Fitted ARIMA models for Acceleration Fatality Functions 


\begin{tabular}{|c|c|c|c|}
\hline $\begin{array}{l}\text { Acceleration } \\
\text { Functions }\end{array}$ & $\begin{array}{c}\text { Fit 1 } \\
\text { (deseasonalized } \\
\text { Auto.arima) } \\
\end{array}$ & $\begin{array}{l}\text { Fit 2(Adjusted } \\
\text { Lag Model) }\end{array}$ & $\begin{array}{c}\text { Fit } 3 \text { (seasonalized } \\
\text { Auto.arima) }\end{array}$ \\
\hline Death_Acceleration1 & $\begin{array}{l}\operatorname{ARIMA}(4,1,5) \\
A I C(-483.27)\end{array}$ & $\begin{array}{l}\operatorname{ARIMA}(7,1,5) \\
\operatorname{AIC}(-556.66)\end{array}$ & $\begin{array}{c}\operatorname{ARIMA}(3,1,5)(0,0,2)[15] \\
A I C(-490.44)\end{array}$ \\
\hline$\frac{d H^{\prime}(z)}{d F^{\prime}(x)}$ & $\begin{array}{l}\operatorname{ARIMA}(1,1,4) \\
A I C(-1087.61)\end{array}$ & $\begin{array}{l}\text { ARIMA }(7,1,4) \\
A I C(-1089.19)\end{array}$ & $\begin{array}{c}\operatorname{ARIMA}(1,1,3)(2,0,0)[15] \\
\text { with drift } \\
A I C(-1092.49)\end{array}$ \\
\hline
\end{tabular}

1 